\title{
Cost-effectiveness of enzyme replacement therapy for Fabry disease
}

\author{
Saskia M Rombach ${ }^{1}$, Carla EM Hollak ${ }^{1}$, Gabor E Linthorst ${ }^{1}$ and Marcel GW Dijkgraaf ${ }^{2^{*}}$
}

\begin{abstract}
Background: The cost-effectiveness of enzyme replacement therapy (ERT) compared to standard medical care was evaluated in the Dutch cohort of patients with Fabry disease.

Methods: Cost-effectiveness analysis was performed using a life-time state-transition model. Transition probabilities, effectiveness data and costs were derived from retrospective data and prospective follow-up of the Dutch study cohort consisting of males and females aged 5-78 years. Intervention with ERT (either agalsidase alfa or agalsidase beta) was compared to the standard medical care. The main outcome measures were years without end organ damage (renal, cardiac en cerebrovascular complications), quality adjusted life years (QALYs), and costs.

Results: Over a 70 year lifetime, an untreated Fabry patient will generate 55.0 years free of end-organ damage (53.5 years in males, 56.9 years in females) and 48.6 QALYs (47.8 in males, 49.7 in females). Starting ERT in a symptomatic patient increases the number of years free of end-organ damage by 1.5 year (1.6 in males, 1.3 in females), while the number of QALYs gained increases by a similar amount (1.7 in males, 1.4 in females). The costs of ERT starting in the symptomatic stage are between $€ 9$ - $€ 10$ million ( $£ 7.9-£ 8.8$ million, \$13.0- \$14.5 million) during a patient's lifetime. Consequently, the extra costs per additional year free of end-organ damage and the extra costs per additional QALY range from $€ 5.5-€ 7.5$ million ( $£ 4.8-£ 6.6$ million, $\$ 8.0-\$ 10.8$ million), undiscounted.
\end{abstract}

Conclusions: In symptomatic patients with Fabry disease, ERT has limited effect on quality of life and progression to end organ damage. The pharmaco-economic evaluation shows that this modest effectiveness drives the costs per QALY and the costs per year free of end-organ damage to millions of euros. Differentiation of patients who may benefit from ERT should be improved to enhance cost-effectiveness.

\section{Background}

Fabry disease (McKusick 301500) is a rare X-linked inherited multisystem lysosomal storage disorder, with an estimated birth prevalence around 1:40,000 [1,2]. Due to a deficiency of alfa-galactosidase A, globotriaosylceramide is stored in various cell types [3]. In hemizygous males, the signs or symptoms include acroparesthesia, inability to sweat, proteinuria, cardiac hypertrophy and cerebral ischemic lesions. Heterozygous females and atypical cases show a more variable and attenuated disease course $[4,5]$. The shortened life expectancy and the morbidity are clearly related to the degree of end-organ damage: progressive renal failure, heart failure, and stroke $[4,6,7]$.

\footnotetext{
*Correspondence: m.g.dijkgraaf@amc.uva.nl

${ }^{2}$ Clinical Research Unit. Academic Medical Centre, PO Box 22660, Amsterdam, DD 1100, The Netherlands

Full list of author information is available at the end of the article
}

In 2002, the EMA approved two recombinant enzymes: agalsidase alfa (Shire HGT, Boston MA, USA) and agalsidase beta (Genzyme Inc, Boston MA, USA). Both received an orphan drug status and have been authorised under "Exceptional Circumstances", which implicates a continuing lack of comprehensive clinical trial data due to the rarity of the disease. With the increasing number of orphan drugs and their extreme costs [8], there is need for more transparency of pricing and reimbursement of orphan drugs, including costeffectiveness analyses [9]. So far, in the UK, effectiveness and cost-effectiveness of enzyme replacement therapies for Fabry disease and mucopolysaccharidosis type 1 have been investigated [10]. Under the assumption that life expectancy and morbidity were completely resolved after treatment, the investigators reported an optimistic incremental cost per additional QALY of 252,000 UK pounds. 
An even lower estimate of the incremental costs per QALY gained with ERT (about US \$300,000) has been calculated by others [11]. Still, giving current market pricing, effectiveness evidence, and efficiency standards for health care, ERT is unlikely to be cost-effective. From a rights-based approach though, it may be argued that individuals are entitled to a decent minimum of health care, including treatment for rare diseases [12].

The Dutch government, by its healthcare insurance board requires the performance of health-economic analyses from a societal perspective for all orphan drugs. The Academic Medical Center in Amsterdam (AMC) was appointed as the coordinating center for the appraisal of enzyme replacement therapy for patients with Fabry disease. A life-time Markov-model was constructed to include the longer term consequences of treatment. The costs per year without end-organ damage and the costs per quality adjusted lifeyear (QALY) constituted the primary outcome measures.

\section{Methods}

\section{Model structure}

A life-time Markov state-transition model of the course of Fabry disease was used to evaluate the costs and effects of ERT against standard medical care. The model comprised eleven disease states including death (Additional file 1 Model):

O No symptoms (no left ventricular hypertrophy, kidney disease, white matter lesions or complications)

O Acroparesthesia (neuropathic pain in the extremities)

O Symptoms (more accurately: clinical signs and/or symptoms of left ventricular hypertrophy, chronic kidney disease stages 1-4, or white matter lesions)

O End stage renal disease (chronic kidney disease stage 5 , dialysis or kidney transplant)

○ Cardiac complication(s) (atrial fibrillation, any other rhythm disturbance needing hospitalization, pacemaker or implantable cardiac defibrillator (ICD) implantation, cardiac congestion for which hospital admittance was needed, myocardial infarction, percutaneous coronary intervention or coronary artery bypass graft)

- Cerebrovascular accident (stroke, as diagnosed by a neurologist)

O End stage renal disease and cardiac complication(s)

O End stage renal disease and cerebrovascular accident

O Cardiac complication(s) and cerebrovascular accident

O End stage renal disease and cardiac complication(s) and cerebrovascular accident

o Death

Typically, patients progress from the asymptomatic state or acroparesthesia state to the symptoms state; from the symptoms state to a single complication state; from a single complication state to a double complication state, and from a double complication state to the triple complication state (Additional file 1 Model). In all states, patients may die. Further, patients with end stage renal disease may return to the symptomatic stage after a kidney transplant (follow-up costs of kidney transplantation were not included). The cycle length of the model was one year.

\section{Model data sources and assumptions}

Data for the estimation of probabilities of transition to the next health state, utilities and costs (see below) were retrospectively and prospectively gathered from the Dutch Fabry cohort (S.M. Rombach et al. OJRD 2013, x:y) including 116 adults and 26 children. Among them, 75 started ERT on indication. Data on disease progression prior to and following the introduction of ERT were gathered from medical chart reviews. Health utility and costs data could only be gathered in the period after the introduction of ERT.

Considering data availability, the limited patient number, and the potential of confounding by indication when contrasting the treatment and no treatment situations, we made several assumptions:

- state-transition probabilities for the natural (untreated) course of Fabry disease are based on the period prior to the introduction of ERT therapy;

- ERT only decreases the probability of progressing to the next disease state;

- health utilities, health care volumes and related costs for treated as well as untreated males and females are similar for patients in the same disease state;

- no distinction was made between agalsidase alfa and agalsidase beta as evidence of superiority of either one of these products is lacking and costs are comparable for the registered dose [13-15].

\section{Transition probabilities}

The yearly transition probabilities for the natural (untreated) course of Fabry disease were calculated by Kaplan-Meier survival analyses. The median (or less if $50 \%$ was not reached) durations to the next states were taken to calculate the transition probabilities, while accounting for the model cycle length of one year [16].

As ERT duration in years rather than treatment with ERT by itself affects the odds of developing major complications (from symptoms to a first complication: odds ratio (OR) 0.82 (95\% CI 0.68-0.96, $\mathrm{p}=0.015$ ); from one complication to the second complication: OR 0.52 (95\% CI 0.31-0.88, p=0.014), S.M. Rombach et al. OJRD 2013, x:y), we calculated the median treatment duration in each disease state and used the odds ratio for treatment duration to calculate the relative risk reduction during that median period; subsequently, we 
calculated the relative risk reduction for each disease state during a single model cycle. The transition probability under ERT was then simply calculated as the yearly transition probability for untreated patients in the same disease state multiplied by 1 minus the relative risk reduction.

If, due to low patient numbers, the probability of dying during the lifetime simulation was lower in the Fabry population than the general population, we used the probability (1-probability of survival) for the general population as published by Statistics Netherlands (CBS, http://statline.cbs.nl/StatWeb/publication/?DM=SLNL\& $\mathrm{PA}=37360$ ned $\& \mathrm{D} 1=0 \& \mathrm{D} 2=\mathrm{a} \& \mathrm{D} 3=\mathrm{a} \& \mathrm{D} 4=60 \& \mathrm{HDR}=\mathrm{G} 1$, $\mathrm{T} \& \mathrm{STB}=\mathrm{G} 3, \mathrm{G} 2 \& V W=\mathrm{T}$, survival rates, as of January $24^{\text {th }} 2011$ ).

The validity of the yearly transition probabilities has been assessed by comparing the model results at fixed time horizons (40, 50, 60, 70 years) with literature data (available upon request) by local experts.

The yearly transition probabilities were assumed to be beta-distributed and 95\% confidence intervals determined by bootstrapping (Additional file 1: Table S1-a to S1-c).

\section{Health outcomes}

Years free of end organ damage reflected years spent in the asymptomatic, acroparesthesia or symptomatic disease states. Health status profiles were gathered quarterly with the EQ-5D quality of life questionnaire; the associated, time trade-off based health utilities [17] were averaged per patient per disease state and, subsequently, per disease state over patients. Given the cycle length of one year in the Markov model, the mean health utility equalled the number of QALYs generated during a single model cycle (Table 1).

\section{Health care volume and costs}

Costs data included the direct and indirect medical costs of health care use as well as the indirect non-medical

Table 1 Mean health utilities per year by disease state cluster and $\mathbf{9 5 \%}$ confidence intervals after boots-trapping

\begin{tabular}{lrccc}
\hline & $\mathbf{N}^{*}$ & $\begin{array}{c}\text { Mean health } \\
\text { utility }\end{array}$ & $\begin{array}{c}\mathbf{9 5 \%} \\
\mathbf{L C L}\end{array}$ & $\begin{array}{c}\mathbf{9 5 \%} \\
\mathbf{U C L}\end{array}$ \\
\hline Asymptomatic & 19 & 0.874 & 0.804 & 0.934 \\
$\begin{array}{l}\text { Acroparesthesia/ } \\
\text { Symptomatic }\end{array}$ & 55 & 0.762 & 0.699 & 0.822 \\
Single complication & 18 & 0.744 & 0.658 & 0.821 \\
Multiple complications & 5 & 0.584 & 0.378 & 0.790 \\
Total & 97 & 0.772 & 0.729 & 0.815 \\
\hline
\end{tabular}

*Patients may contribute to more than one disease state.

Given low patient numbers in the more progressive disease states, results were clustered by four states: asymptomatic, acroparesthesia/symptomatic, single complication, multiple complications. LCL: lower confidence limit. UCL: upper confidence limit. costs of sick leave. The resource use data from the AMC were linked to available real unit costs from the AMC hospital ledger [18] (see Table 2). Unit costs were priceindexed for the year 2009.

The mean yearly costs of ERT per patient were determined for a patient with an average weight of $70 \mathrm{~kg}$ (priceindexed for 2009: €200,503 for agalsidase alpha and $€ 199,452$ for agalsidase beta), or about $€ 200,000$ yearly. The costs of AMC hospital care were averaged per patient per disease state per year, and subsequently, the mean yearly AMC costs per disease state overall (Additional file 1: Table S2). All other used health care costs and production loss were derived from the patient by quarterly disseminated questionnaires and linked to the appropriate unit costs in Euros from the most recent Dutch costing manual (Additional file 1: Table S3 and S4). The specialist physician costs are included in the hospital costs. For each hour of production loss irrespective of gender, age, or disease state the same unit cost was applied ( $€ 30$, see Table 2) (Additional file 1: Table S5). No account was given of lost productivity during working hours. Additional file 1: Table S5 shows the mean yearly costs of production loss by disease state cluster (Additional file 1: Table S5). Ethical approval was requested at the institutional review board, METC AMC. The institutional review board stated that ethical approval was not required.

\section{Analysis}

The model was run from a lifetime perspective, starting asymptomatically at birth until the age of 70 years or death. Hypothetical cohorts of treated and untreated male and female patients were compared for both primary outcomes: costs per year without end-organ damage and costs per QALY. Incremental lifetime costeffectiveness ratios were calculated by dividing the lifetime costs difference by the difference in lifetime years free of end-organ damage or by the difference in lifetime QALYs gained.

In the base case scenario, patients (males and females at a 1:1 ratio) entered the model at birth; ERT was initiated when symptoms developed; the costs only included the total direct and indirect medical costs; no discounting of effects or costs was performed. Univariate sensitivity analyses have been restricted to the choice of discount rate to account for time preference (discounting of effects by $1.5 \%$ and costs by $4 \%$ instead of no discounting) [22], to the unit cost of ERT per year (minus $€ 50,000$ ), and to a Dutch time trade-off based health utility algorithm [23].

We performed a two-stage Monte Carlo simulation of 1,000 second-order draws from the beta-distributed yearly transition probabilities in the Markov model to represent parameter uncertainty with each single draw including 10 first-order trials to represent patient heterogeneity. For all 1,000 runs in the simulation, the net 
Table 2 Dutch unit costs $(€)$ for resources used

\begin{tabular}{|c|c|c|}
\hline Resource & $\begin{array}{l}\text { Unit costs in } \\
2009(€) \#\end{array}$ & Source \\
\hline \multicolumn{3}{|l|}{ Inpatient hospital day } \\
\hline AMC & $596-1,036$ & AMC hospital ledger\#\# \\
\hline Elsewhere* & 457 & Dutch costing manual** \\
\hline Inpatient hospital ICU day & 2,183 & Dutch costing manual \\
\hline \multicolumn{3}{|l|}{$\begin{array}{l}\text { In-hospital day-care } \\
\text { treatment }\end{array}$} \\
\hline AMC & $274-845$ & AMC hospital ledger \\
\hline Elsewhere & 251 & Dutch costing manual \\
\hline $\begin{array}{l}\text { Agalsidase } \alpha / \beta^{* * *} \text { per } \\
\text { patient per year }\end{array}$ & 200,000 & $\begin{array}{l}\text { Report manufacturer } \\
\text { 2010; z-index } 2007\end{array}$ \\
\hline Kidney dialysis per year & 60,000 & {$[19,20]$} \\
\hline \multicolumn{3}{|l|}{ Kidney transplantation } \\
\hline first year & 60,000 & {$[19,20]$} \\
\hline follow-up per year & not included & \\
\hline $\begin{array}{l}\text { Other diagnostic and } \\
\text { therapeutic procedures }\end{array}$ & Various & AMC hospital ledger \\
\hline \multicolumn{3}{|l|}{ Outpatient hospital visit } \\
\hline AMC & $90-460$ & AMC hospital ledger \\
\hline Elsewhere* & 72 & Dutch costing manual \\
\hline \multicolumn{3}{|l|}{ Out-of-hospital visit } \\
\hline General practitioner & 28 & Dutch costing manual \\
\hline Physiotherapist & 36 & Dutch costing manual \\
\hline $\begin{array}{l}\text { Psychiatrist/ } \\
\text { psychologistt }\end{array}$ & 91.5 & Dutch costing manual \\
\hline $\begin{array}{l}\text { Occupational } \\
\text { physician/othert† }\end{array}$ & 26 & AMC hospital ledger \\
\hline Social worker & 65 & Dutch costing manual \\
\hline Alternative healer & 60 & Expert opiniont†† \\
\hline Productivity loss per hour^ & 30 & Dutch costing manual \\
\hline
\end{tabular}

\# In case of different base years the general price index figures from the Dutch costing manual 2010 have been used to derive 2009 estimates. \#\# Unit costs from the AMC hospital ledger for Fabry patients include the costs of top referent health care. * Weighted unit cost based on $88 \%$ general and $12 \%$ academic inpatient days. ${ }^{* *}$ Unit costs from the most recent Dutch costing manual [21]. *** Weighted mean costs of therapy per patient of $70 \mathrm{~kg}$ per year. The costs per patient per year of full treatment amount to $€ 198,640$ for agalsidase- $\alpha$ and $€ 201,346$ (price-indexed for 2009) for agalsidase- $\beta$. $\dagger$ Weighted unit cost based on the assumption of 50\%-50\% distribution of visits over psychiatrists $(€ 103)$ and psychologists $(€ 80)$ respectively. t† Out-of -hospital visit to other care givers are assigned the lowest unit costs among the caregivers, i.e. the occupational physician. $+1+$ The Nederlandse Mededingings Autoriteit prohibits the use of an advised tariff. Unit costs per consultation may vary considerably, depending on the type of alternative healer. As a proxy, the reported unit cost of $€ 60$ per visit is based on an indexed derivation of the advised 2000 tariff for an acupuncturist. ^ Mean unit costs per hour across gender and age groups.

monetary benefits of ERT versus standard medical care were calculated for willingness-to-pay levels ranging from $€ 20,000$ to $€ 10,000,000$ per QALY with the results summarized in a cost-effectiveness acceptability curve for ERT.
In contrast with the base case scenario we additionally ran six alternative scenarios:

(1) the start of ERT in the model at the time symptoms develop is delayed until the age of 40 years, which reflects the mean age of treatment start in the Dutch Fabry cohort prior to 2010.

(2) a lower quality of life for untreated patients compared to treated patients in the same disease state with a mean difference in health utility of 0.1 [24].

(3) course of disease in patients with the classical phenotype only, without the atypical cases with a more attenuated disease course [25]. Atypical patients included patients with the $\mathrm{R} 112 \mathrm{H}$ and $\mathrm{P} 60 \mathrm{~L}$ substitutions or patients with intermediate levels of lysoGb3 (S.M. Rombach et al. OJRD 2013, x:y).

(4) the natural course of disease in case all patients were treated with ACE-inhibitors or angiotensinreceptor blockers (which is frequent co-medication). The potential beneficial effects are extrapolated from studies in patients at high risk for cardiovascular events [26-30].

(5) no ERT for patients in the second complication groups, as previous studies have doubted the beneficial effect of ERT in more severely affected patients.

(6) adding indirect non-medical costs of production loss to the direct and indirect medical costs, assuming that these costs originate not before the $18^{\text {th }}$ year of a patient's lifetime.

\section{Results}

\section{Base case scenario}

Over a 70 years lifetime and undiscounted, an untreated Fabry patient generates 55.0 years free of end-organ damage and 48.6 QALYs. Starting ERT in a symptomatic patient increases the number of years free of end-organ damage by 1.5 years (1.6 for males, 1.3 for females), while the number of QALYs gained increases by 1.6 QALY (1.7 for males, 1.4 for females). Table 3 shows the discounted (at 1.5\%) and undiscounted incremental lifetime effects of ERT, overall and by gender.

The undiscounted costs of ERT starting in the symptomatic stage amount to $€ 9.9$ million ( $€ 9.6 \mathrm{M}$ for males, $€ 10 \mathrm{M}$ for females) during a patient's lifetime against $€ 0.271$ million ( $€ 0.273 \mathrm{M}$ for males, $€ 0.268 \mathrm{M}$ for females) for standard medical care, the difference being €9.6 million (€9.3 M for males, $€ 9.8 \mathrm{M}$ for females). Table 4 shows the discounted (at 4\%) and undiscounted incremental lifetime costs of ERT, overall and by gender.

Table 5 shows the discounted and undiscounted incremental lifetime cost-effectiveness ratios (ICER), overall and by gender. 
Table 3 Discounted and undiscounted incremental lifetime effects of ERT versus no ERT treatment, overall and by gender (YFEOD: years free of end-organ damage; QALYs: quality adjusted life-years)

\begin{tabular}{|c|c|c|c|c|c|c|}
\hline & \multicolumn{3}{|c|}{ Discount rate $1.5 \%$} & \multicolumn{3}{|c|}{ Discount rate $0 \%$} \\
\hline & ERT & No ERT & Difference & ERT & No ERT & Difference \\
\hline \multicolumn{7}{|l|}{ ALL } \\
\hline YFEOD & 36.9 & 36.1 & 0.7 & 56.5 & 55.0 & 1.5 \\
\hline QALYS & 32.1 & 31.3 & 0.7 & 50.2 & 48.6 & 1.6 \\
\hline \multicolumn{7}{|l|}{ MALES } \\
\hline YFEOD & 36.2 & 35.4 & 0.8 & 55.1 & 53.5 & 1.6 \\
\hline QALYS & 31.7 & 30.9 & 0.8 & 49.5 & 47.8 & 1.7 \\
\hline \multicolumn{7}{|l|}{ FEMALES } \\
\hline YFEOD & 37.7 & 37.1 & 0.6 & 58.2 & 56.9 & 1.3 \\
\hline QALYS & 32.6 & 31.9 & 0.7 & 51.1 & 49.7 & 1.4 \\
\hline
\end{tabular}

The table shows that the ICER based on years free of end-organ damage equals $€ 6.6$ million ( $€ 5.9 \mathrm{M}$ for males and $€ 7.5 \mathrm{M}$ for females); the incremental costs per QALY gained equal $€ 6.1$ million ( $€ 5.5 \mathrm{M}$ for males, $€ 7.0 \mathrm{M}$ for females).

\section{Scenario analyses}

Table 6 shows the discounted and undiscounted incremental lifetime ICERs for years free of end-organ damage and for QALYs respectively under different scenarios.

If ERT is not initiated before the age of 40 , both, lifetime costs and effects decrease, but to a different extent, resulting in increased undiscounted ICERs, especially in case of the incremental costs per extra year free of endorgan damage. If ERT not only slows disease progression, but also results in a higher health utility of 0.1 when treated, the undiscounted ICER based on QALYs drops by $80 \%$ to $€ 1.2$ million. ERT in classically affected patients only results in $5 \%$ to $8 \%$ lower ICERs compared to the base case. The impact of additional treatment with ACE-ARB minimizes the value of ERT as is demonstrated by the increased ICERs compared with the base case scenario. The final two scenarios, not treating patients with more advanced disease or adding the indirect costs of production loss to the total medical costs, marginally affect the ICERs. In all scenarios, the
ICERs were more favourable for males than for females (data not shown).

\section{Sensitivity analyses}

Differential discounting of effects (at 1.5\%) and costs (at $4 \%$ ) improves the undiscounted ICERs for the base case scenario as well as for scenarios 3 to 6 by $48-49 \%$ in case of years free of end-organ damage and by 45$46 \%$ in case of QALYs. Application of discounting in the scenario start ERT at the age of 40 decreases the undiscounted ICERs by $72 \%$. Discounting decreases the incremental costs per extra QALY in the scenario with a lower health utility when untreated by $58 \%$.

A 25\% reduction of the yearly cost of ERT from $€ 200,000$ to $€ 150,000$ demonstrates the dominance of this cost component: the incremental cost-effectiveness ratios decrease by a similar margin. For instance, the extra costs per additional QALY for a typical patient, a male patient and a female patient amount to $€ 4,549,987$, $€ 4,089,674$ and $€ 5,217,573$ respectively against $€ 6.1$ million, $€ 5.5$ million and $€ 7.0$ million in the base case scenario.

Mean health utilities by disease state based on preferences from the Dutch general populations are non-significantly higher than the UK based data [17], with lower losses in health utility during disease progression. Hence, slowing disease progression results in less QALYs to be gained, if Dutch preferences were to be used instead. Consequently, the ICERs lie above the UK based values reported in this paper.

Figure 1 shows the cost-effectiveness acceptability curves of ERT against standard medical care for various levels of the willingness-to-pay per QALY and in absence of discounting. It shows that the probability of ERT being an efficient intervention is near zero when more conventional willingness-to-pay values up to $€ 100,000$ are considered and does not exceed 0.5 for values below $€ 10$ million.

\section{Discussion}

\section{Summary of major findings}

The model demonstrated small gains in effectiveness with 1.5 extra years free of end-organ damage and 1.6 QALYs gained (or both 0.7, discounted) for treatment with ERT. The extra lifetime costs of ERT compared

Table 4 Discounted and undiscounted incremental lifetime total medical costs of ERT versus no ERT treatment, overall and by gender

\begin{tabular}{|c|c|c|c|c|c|c|}
\hline & \multicolumn{3}{|c|}{ Discount rate $4 \%$} & \multicolumn{3}{|c|}{ Discount rate $0 \%$} \\
\hline & ERT & No ERT & Difference & ERT & No ERT & Difference \\
\hline ALL & $€ 2,504,727$ & $€ 83,772$ & $€ 2,420,956$ & $€ 9,918.352$ & $€ 270,964$ & $€ 9.647,388$ \\
\hline MALES & $€ 2,433,824$ & $€ 85,305$ & $€ 2,348,519$ & $€ 9,615,920$ & $€ 272,892$ & $€ 9,343,028$ \\
\hline FEMALES & $€ 2,516,273$ & $€ 81,624$ & $€ 2,434,649$ & $€ 10,056,623$ & $€ 267,517$ & $€ 9,789,106$ \\
\hline
\end{tabular}


Table 5 Discounted and undiscounted incremental lifetime cost-effectiveness ratio's, overall and by gender

\begin{tabular}{|c|c|c|}
\hline & $\begin{array}{r}\text { Discount rate at } 1.5 \% \\
\text { for effects and } 4 \% \text { for } \\
\text { costs }\end{array}$ & $\begin{array}{r}\text { Discount rate at } \\
0 \% \text { for effects } \\
\text { and costs }\end{array}$ \\
\hline \multicolumn{3}{|l|}{ ALL } \\
\hline $\begin{array}{l}\text { Incremental costs per } \\
\text { extra year free of end- } \\
\text { organ damage }\end{array}$ & $€ 3,318,239$ & $€ 6,560,885$ \\
\hline $\begin{array}{r}\text { Incremental costs per } \\
\text { QALY gained }\end{array}$ & $€ 3,282,252$ & $€ 6,065,529$ \\
\hline \multicolumn{3}{|l|}{ MALES } \\
\hline $\begin{array}{l}\text { Incremental costs per } \\
\text { extra year free of end- } \\
\text { organ damage }\end{array}$ & $€ 2,982,022$ & $€ 5,917,091$ \\
\hline $\begin{array}{r}\text { Incremental costs per } \\
\text { QALY gained }\end{array}$ & $€ 2,947,380$ & $€ 5,451,797$ \\
\hline \multicolumn{3}{|l|}{ FEMALES } \\
\hline $\begin{array}{l}\text { Incremental costs per } \\
\text { extra year free of end- } \\
\text { organ damage }\end{array}$ & $€ 3,797,767$ & $€ 7,527,013$ \\
\hline $\begin{array}{r}\text { Incremental costs per } \\
\text { QALY gained }\end{array}$ & $€ 3,742,702$ & $€ 6,955,612$ \\
\hline
\end{tabular}

with standard medical care amounted to $€ 9.65$ million per patient (or $€ 2.42$ million, discounted). The related incremental cost-effectiveness ratios ranged from $€ 3.2$ million (discounted) to $€ 6.5$ million (no discounting).

The sensitivity and scenario analyses revealed that these cost-effectiveness ratios could be substantially reduced by lowering the high costs of the drug itself (near proportional impact) or a modest health gain of 0.1 QALY per year in treated patients (minus $80 \%$ ). To a lesser extent, patient selection optimizes the ICERs, e.g. classically affected patients with a more severe disease course [25].

The probabilistic sensitivity analysis showed that the uncertainties concerning the estimated transition probabilities only affect health care policy making substantially as of willingness-to-pay values of $€ 1$ million per QALY or above.
On the other hand, the additional value of ERT could easily be nullified, if ACE-ARB for the reduction of proteinuria reduces the risk on cardiovascular complications equally well in Fabry patients as in other high risk populations [31,32]. This is mostly explained by the assumption that treatment with ACE-ARB alone would be as effective as ERT for risk reduction of a first complication (see Additional file 2). Whether this assumption is valid, is a research area of interest.

The most cost-effective scenario is early treatment of a male patient with a classical phenotype and discarding additional beneficial effect of ACE-ARB. In contrast, in milder affected patients, such as women and atypical phenotypes, the gain in QALYs with ERT is only modest. At equal costs of treatment, cost-effectiveness is consequently less. It needs to be emphasized that differentiating by gender is an oversimplification: some females may be as severely affected as males and thus could equally benefit from therapy.

Our assumption that the health utilities, health care volume and the related costs other than the costs of ERT medication itself were similar for treated and untreated patients in the same disease state should be considered as a study limitation. The scenario of a lower health utility during the natural course demonstrated that this may lead to an underestimation of the value of ERT. Actually, it would be of the utmost importance to accurately assess the difference in health utility between patients on ERT and patients not on ERT in the "real world", without being hampered by confounding by indication. Unfortunately, there is a lack of opportunity in this respect.

Another study limitation was the exclusion of costs of follow-up related to a kidney transplant in particular; only the costs of a kidney transplant during its first year were incorporated. Including these follow-up costs would have increased the structural complexity of the memory-less Markov model considerably without - in view of the small number of Dutch Fabry patients receiving a kidney transplant - meaningful consequences in terms of health policy.

Table 6 Scenario-analyses*: discounted and undiscounted incremental lifetime cost- effectiveness ratios

\begin{tabular}{|c|c|c|c|c|}
\hline & \multicolumn{2}{|c|}{$\begin{array}{l}\text { ICER based on years free of end-organ damage, } \\
\text { in } €\end{array}$} & \multicolumn{2}{|c|}{ ICER based QALYs, in $€$} \\
\hline & Discounted & Undiscounted & Discounted & Undiscounted \\
\hline Base case typical patient & $3,318,239$ & $6,560,885$ & $3,282,252$ & $6,065,529$ \\
\hline 1. start ERT at 40 years & $3,662,891$ & $12,996,662$ & $2,158,245$ & $7,637,076$ \\
\hline 2. lower QoL during the natural course & - & - & 509,719 & $1,226,674$ \\
\hline 3. course of disease in patients with the classical phenotype only & $3,274,869$ & $6,280,356$ & $3,015,385$ & $5,575,064$ \\
\hline 4. ACE-ARB during natural course & $293,213,929$ & $566,675,324$ & $11,559,105$ & $21,223,686$ \\
\hline 5. No ERT in case of 2 complications & $3,307,363$ & $6,529,644$ & $3,271,494$ & $6,036,644$ \\
\hline 6. including indirect costs of production loss & $3,320,374$ & $6,568,971$ & $3,284,265$ & $6,073,006$ \\
\hline
\end{tabular}

* Given a 1:1 male to female ratio. 


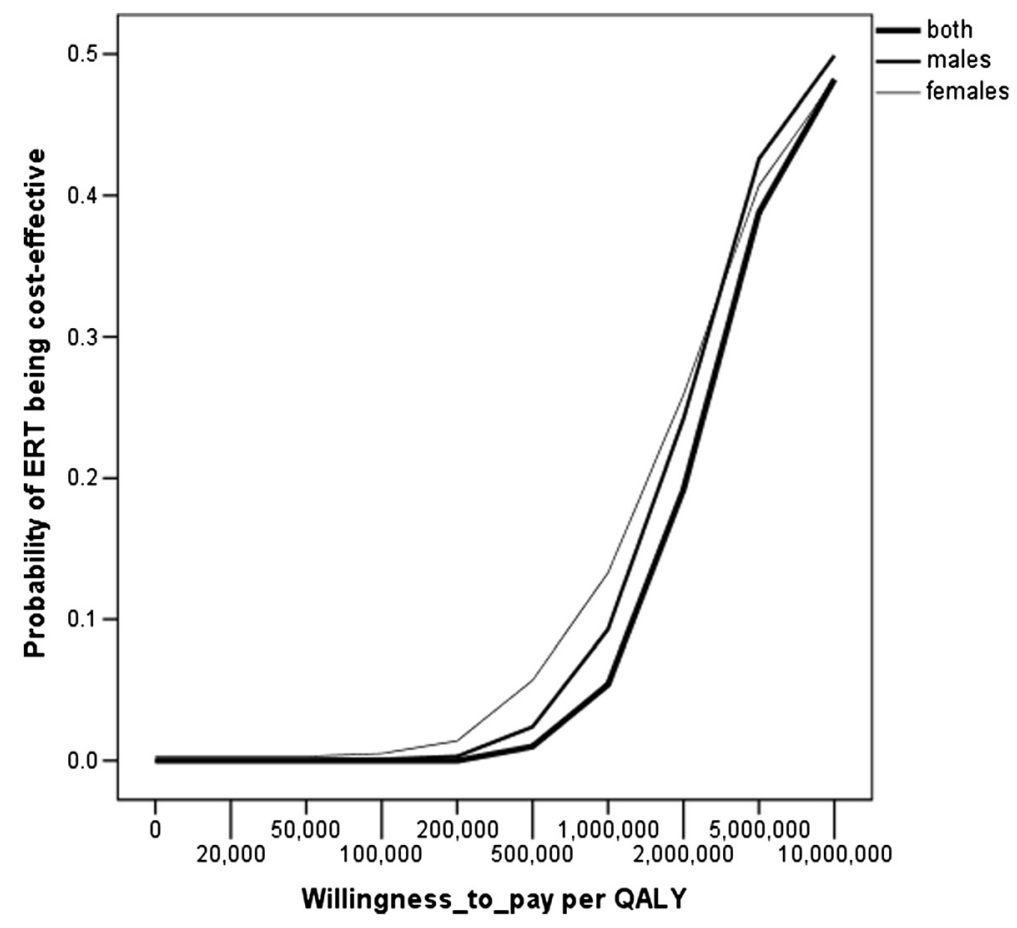

Figure 1 Cost-effectiveness acceptability curves of ERT versus no treatment for various levels of willingness-to-pay per QALY after 1,000 runs in a Monte Carlo simulation of beta-distributed state transition probabilities. The proportions of net monetary benefits larger than zero for these willingness-to-pay levels represent the probabilities of ERT being cost-effective in comparison with standard medical care and are reported in the cost-effectiveness acceptability curve.

When comparing the treatment effect as determined in the Dutch Fabry cohort to the results of the only randomized-controlled trial on development of endorgan complications available, we notice a similar risk reduction (for one complication, developing a second complication) [32]. This supports the validity of the estimated treatment effect used in the model.

\section{Implications and recommendations for future studies}

The present study highlights the enormous costs associated with orphan drugs as was recently also pointed out by others $[8,9]$. Compared to previously reported international data on costs per QALY, ranging from US $\$ 300,000$ to UK $£ 250,000$ per QALY, the present results should put (i) the high unit costs of ERT itself, (ii) the process of drug development with perhaps a lack of sufficient early assessment of a drug's clinical potential, and (iii) the cooperation between manufacturers and governmental agencies on the agenda.

As ERT itself is costly, the ICERs are reported in millions and are not coming near to thresholds that might be considered affordable from a societal perspective [33]. Recently, a health technology assessment in the UK showed that at least 3.6 discounted QALYs for an adult patient with Fabry disease are needed each year for ERT to be costeffective, considering a willingness-to-pay of $£ 30000$ [34].
Based on the incremental lifetime total medical costs data generated by the Markov model (see Table 4), a willingness-to-pay per QALY in the Netherlands of $€ 80$ 000 , and a treatment window of about 46 years between the onset of symptoms and death in the natural history cohort, at least 0.65 discounted and 2.6 undiscounted QALYs would have to be generated each year under ERT treatment. It is clear that such yearly gain is unrealistic, which makes it understandable, if one considers performing a cost-effectiveness or cost-utility analysis infeasible [34].

Based on the data on disease progression while simultaneously aiming for cost containment, new therapeutic guidelines should therefore be developed to differentiate patients who may benefit from ERT from those who probably will not. Incorporating findings on associations between ERT and key disease progression markers in Fabry disease from the abovementioned health technology assessment in the UK, especially with regard to cardiac and renal manifestations, may prove highly valuable in this respect [34].

At this time no data exist that support the idea of agalsidase alfa being superior to agalsidase beta, or viceversa. It was beyond the scope of this study to address this.

Pre-symptomatic initiation of ERT may improve costeffectiveness by prevention of complications that impact 
on quality of life but there are no data available yet to evaluate this scenario.

\section{Conclusion}

In conclusion, this study showed that the affordability of ERT of Fabry disease remains at stake. The modest effectiveness drives the costs per QALY and even the costs per year free of end-organ damage to numbers expressed in millions of euros. New therapeutic guidelines should be developed to differentiate high responders from low or no responders to ERT, diagnostic procedures should be improved, and the add-on value of ERT relative to the effect of ACE-ARB should be assessed.

\section{Additional file}

Additional file 1: Model Markov model for Fabry disease. Model

structure for bottom half (ERT treatment) is identical. In all Markov states, patients may die (not shown). Table S1-a. Yearly beta-distributed statetransition probabilities for untreated and treated males. Table S1-b. Yearly beta-distributed state-transition probabilities for untreated and treated females. Table S1-c. Yearly beta-distributed state-transition probabilities for untreated and treated males and females. Table S2. Mean yearly numbers and costs of diagnostic and therapeutic procedures* in the AMC. Clustering of disease states was necessary because of low patient numbers. LCL: lower confidence limit. UCL: upper confidence limit. Table S3. Mean yearly numbers and costs of (ICU) inpatient days in hospitals other than the AMC. Costs were derived from the quarterly disseminated patient questionnaires and averaged per patient per disease state cluster, multiplied by four to arrive at yearly mean estimates per patient per disease state cluster, and subsequently, averaged per disease state cluster. LCL: lower confidence limit. UCL: upper confidence limit. Table S4. Mean yearly numbers and costs of various out-of-hospital consultations by disease state cluster. First, costs per individual patient per disease state per year were calculated; subsequently, the total average per patient per disease state was calculated. LCL: lower confidence limit. UCL: upper confidence limit. Table S5. Mean yearly indirect costs of sick leave by disease state cluster. The overall mean number of working hours per working day and overall mean number of working days per week for patients with paid work were calculated. For each patient with a paid job the individual mean volume of sick leave in days per fortnight was calculated over available repeated measurements and per disease state. The resulting individual mean volume was multiplied by 26 and by the overall mean number of hours per working day for patients with a paid job to arrive at yearly mean production loss estimates per patient per disease state. For patients with a permanent sick leave because of Fabry disease a yearly volume of production loss was defined based on the overall mean number of working hours per working day and overall mean number of working days per week for patients with paid work. For patients without paid work for reasons other than Fabry disease a zero volume of production loss was assumed. LCL: lower confidence limit. UCL: upper confidence limit.

Additional file 2: APPENDIX. The cost-effectiveness and cost-utility model.

\section{Competing interests}

CEH and GEL received reimbursement of expenses and honoraria for lectures on the management of lysosomal storage diseases from Genzyme Corporation, Shire, Actelion and Amicus Therapeutics. All honoraria are donated to the Gaucher Stichting, a national foundation that supports research in the field of lysosomal storage disorders. SMR and MGD declare that they have no competing interests.

\section{Authors' contributions}

CEM and MGD initiated the study. SMR coordinated the study. SMR and GEL participated in the data collection. All authors were involved in the design of the study. SMR, CEM and MGD contributed to the analysis. All authors were involved in writing the report. All authors read and approved the final manuscript.

\section{Funding}

This study was supported by a grant from the Ministry of Health (ZonMW). Researchers worked independently from the funders. The funding source had no involvement in study design; in the collection, analysis, and interpretation of data; in the writing of the report; and in the decision to submit an article for publication.

\section{Author details}

'Department of Internal Medicine, Division of Endocrinology and Metabolism, Academic Medical Centre, PO Box 22660, Amsterdam, DD 1100 , The Netherlands. ${ }^{2}$ Clinical Research Unit. Academic Medical Centre, PO Box 22660, Amsterdam, DD 1100, The Netherlands.

Received: 23 October 2012 Accepted: 9 February 2013 Published: 19 February 2013

\section{Reference}

1. Hwu WL, Chien YH, Lee NC, Chiang SC, Dobrovolny R, Huang AC, et al Newborn screening for Fabry disease in Taiwan reveals a high incidence of the later-onset GLA mutation c.936+919 G>A (IVS4+919 G>A). Hum Mutat 2009, 30(10):1397-1405.

2. Spada M, Pagliardini S, Yasuda M, Tukel T, Thiagarajan G, Sakuraba H, et al: High incidence of later-onset fabry disease revealed by newborn screening. Am J Hum Genet 2006, 79(1):31-40.

3. Desnick RJ, loannou YA, Eng CM: alpha-Galactosidase A deficiency: Fabry disease. In The metabolic and molecular bases of inherited disease, Volume 3. 8th edition. Edited by Scriver CR, Beaudet AL, Sly WS, Valle D.; 2001:3733-3774.

4. MacDermot KD, Holmes A, Miners AH: Anderson-Fabry disease: clinical manifestations and impact of disease in a cohort of 60 obligate carrier females. J Med Genet 2001, 38(11):769-775.

5. Whybra C, Kampmann C, Willers I, Davies J, Winchester B, Kriegsmann J, et al: Anderson-Fabry disease: clinical manifestations of disease in female heterozygotes. J Inherit Metab Dis 2001, 24(7):715-724

6. MacDermot KD, Holmes A, Miners AH: Anderson-Fabry disease: clinical manifestations and impact of disease in a cohort of 98 hemizygous males. J Med Genet 2001, 38(11):750-760.

7. Vedder $\mathrm{AC}$, Linthorst $\mathrm{GE}$, van Breemen MJ, Groener JE, Bemelman FJ, Strijland $A$, et al: The Dutch Fabry cohort: diversity of clinical manifestations and Gb3 levels. J Inherit Metab Dis 2007, 30(1):68-78.

8. Roos JC, Hyry HI, Cox TM: Orphan drug pricing may warrant a competition law investigation. BMJ 2010, 341:c6471.

9. Simoens S: Pricing and reimbursement of orphan drugs: the need for more transparency. Orphanet J Rare Dis 2011, 6:42.

10. Connock M, Juarez-Garcia A, Frew E, Mans A, Dretzke J, Fry-Smith A, et al: A systematic review of the clinical effectiveness and cost-effectiveness of enzyme replacement therapies for Fabry's disease and mucopolysaccharidosis type 1. Health Technol Assess 2006, 10(20):iii-113.

11. Moore DF, Ries M, Forget EL, Schiffmann R: Enzyme replacement therapy in orphan and ultra-orphan diseases: the limitations of standard economic metrics as exemplified by Fabry-Anderson disease. Pharmacoeconomics 2007, 25(3):201-208.

12. Hughes DA, Tunnage B, Yeo ST: Drugs for exceptionally rare diseases: do they deserve special status for funding? QJM 2005, 98(11):829-836.

13. Blom D, Speijer D, Linthorst GE, Donker-Koopman WG, Strijland A, Aerts JM Recombinant enzyme therapy for Fabry disease: absence of editing of human alpha-galactosidase A mRNA. Am J Hum Genet 2003, 72(1):23-31.

14. Vedder AC, Linthorst GE, Houge G, Groener JE, Ormel EE, Bouma BJ, et al: Treatment of Fabry disease: outcome of a comparative trial with agalsidase alfa or beta at a dose of $0.2 \mathrm{mg} / \mathrm{kg}$. PLoS One 2007, 2(7):e598.

15. Sirrs S, Clarke JT, Bichet DG, Casey R, Lemoine K, Flowerdew G, et al: Baseline characteristics of patients enrolled in the Canadian Fabry Disease Initiative. Mol Genet Metab 2010, 99(4):367-373. 
16. Briggs A, Sculpher M: An introduction to Markov modelling for economic evaluation. Pharmacoeconomics 1998, 13(4):397-409.

17. Dolan P: Modeling valuations for EuroQol health states. Med Care 1997, 35(11):1095-1108

18. Asselman F: Unit costs in hospitals. Meppel: Bohn Stafleu van Loghum; 2008.

19. van den Ham ECH: Body composition and exercise intolerance in renal transplant patients: the response to exercise training. Maastricht: University of Maastricht, dissertation; 2006.

20. Muntinghe FL, Vegter S, Verduijn M, Boeschoten EW, Dekker FW, Navis G, et al: Using a genetic, observational study as a strategy to estimate the potential cost-effectiveness of pharmacological CCR5 blockade in dialysis patients. Pharmacogenet Genomics 2011, 21(7):417-425.

21. Hakkaart-van Roijen L, Tan S, Bouwmans CAM: Manual for cost research; methods and unitcosts for economic evaluations in health care. Amsterdam: Dutch College of Health Care Insurance, Actualized version; 2010.

22. Brouwer WB, Niessen LW, Postma MJ, Rutten FF: Need for differential discounting of costs and health effects in cost effectiveness analyses. BMJ 2005, 331(7514):446-448.

23. Lamers LM, Stalmeier PF, McDonnell J, Krabbe PF, van Busschbach JJ: Measuring the quality of life in economic evaluations: the Dutch EQ-5D tariff. Ned Tijdschr Geneeskd 2005, 149(28):1574-1578.

24. Hoffmann B, de Garcia LA, Mehta A, Beck M, Widmer U, Ricci R: Effects of enzyme replacement therapy on pain and health related quality of life in patients with Fabry disease: data from FOS (Fabry Outcome Survey). J Med Genet 2005, 42(3):247-252.

25. Aerts JM, Groener JE, Kuiper S, Donker-Koopman WE, Strijland A, Ottenhoff $\mathrm{R}$, et al: Elevated globotriaosylsphingosine is a hallmark of Fabry disease. Proc Natl Acad Sci USA 2008, 105(8):2812-2817.

26. Yusuf S, Sleight P, Pogue J, Bosch J, Davies R, Dagenais G: Effects of an angiotensin-converting-enzyme inhibitor, ramipril, on cardiovascular events in high-risk patients. The heart outcomes prevention evaluation study investigators. N Engl J Med 2000, 342(3):145-153.

27. HOPE investigators: Effects of ramipril on cardiovascular and microvascular outcomes in people with diabetes mellitus: results of the HOPE study and MICRO-HOPE substudy. Heart outcomes prevention evaluation study investigators. Lancet 2000, 355(9200):253-259.

28. Dagenais GR, Yusuf S, Bourassa MG, Yi Q, Bosch J, Lonn EM, et al: Effects of ramipril on coronary events in high-risk persons: results of the heart outcomes prevention evaluation study. Circulation 2001, 104(5):522-526.

29. Schadlich PK, Brecht JG, Rangoonwala B, Huppertz E: Cost effectiveness of ramipril in patients at high risk for cardiovascular events: economic evaluation of the HOPE (heart outcomes prevention evaluation) study for germany from the statutory health insurance perspective. Pharmacoeconomics 2004, 22(15):955-973.

30. Hackam DG, Duong-Hua ML, Mamdani M, Li P, Tobe SW, Spence JD, et al: Angiotensin inhibition in renovascular disease: a population-based cohort study. Am Heart J 2008, 156(3):549-555.

31. Germain DP, Waldek S, Banikazemi M, Bushinsky DA, Charrow J, Desnick RJ, et al: Sustained, long-term renal stabilization after 54 months of agalsidase beta therapy in patients with Fabry disease. J Am Soc Nephrol 2007, 18(5):1547-1557.

32. Banikazemi M, Bultas J, Waldek S, Wilcox WR, Whitley CB, McDonald M, et al: Agalsidase-beta therapy for advanced Fabry disease: a randomized trial. Ann Intern Med 2007, 146(2):77-86.

33. Simoens S: Biosimilar medicines and cost-effectiveness. Clinicoecon Outcomes Res 2011, 3:29-36.

34. Wyatt K, Henley W, Anderson L, Anderson R, Nikolaou V, Stein K, et al: The effectiveness and cost-effectiveness of enzyme and substrate replacement therapies: a longitudinal cohort study of people with lysosomal storage disorders. Health Technol Assess 2012, 16(39):1-543.

doi:10.1186/1750-1172-8-29

Cite this article as: Rombach et al: Cost-effectiveness of enzyme replacement therapy for Fabry disease. Orphanet Journal of Rare Diseases 2013 8:29.

\section{Submit your next manuscript to BioMed Central and take full advantage of:}

- Convenient online submission

- Thorough peer review

- No space constraints or color figure charges

- Immediate publication on acceptance

- Inclusion in PubMed, CAS, Scopus and Google Scholar

- Research which is freely available for redistribution

Submit your manuscript at www.biomedcentral.com/submit
Biomed Central 